\title{
Little-Parks effect in a superconducting loop with magnetic dot
}

\author{
D. S. Golubović*, W. V. Pogosov, M. Morelle and V. V. Moshchalkov \\ Nanoscale Superconductivity and Magnetism Group, \\ Laboratory for Solid State Physics and Magnetism, \\ K. U. Leuven, Celestijnenlaan 200 D, B-3001 Leuven, Belgium
}

\begin{abstract}
We have studied the nucleation of superconductivity in a mesoscopic Al loop, enclosing magnetic dot with the perpendicular magnetization. The superconducting phase boundary $T_{c}(B)$, determined from transport measurements, is asymmetric with respect to the polarity of an applied magnetic field. The maximum critical temperature has been found for a finite applied magnetic field, which is antiparallel to the magnetization of the dot. Theoretical phase boundary shows a good agreement with the experimental data.

PACS numbers: 74.78.Na,73.23.-b,74.25.Dw
\end{abstract}

A great deal of experimental and theoretical work has been devoted to the investigation of mesoscopic superconductors (e.g. ref. 1, 2] and references therein). Over the past few years hybrid superconductor/ferromagnet systems have attracted a lot of attention $3,4,5,6,7,8,9,10,11,12]$. It has been revealed that a magnetic dot, embedded into a superconductor, attracts a vortex, resulting in an enhanced pinning when the magnetic moment $m$ of magnetic dot and an applied magnetic field $B$ have parallel orientation 1, 11, 13. Moreover, it was recently demonstrated that perpendicularly magnetized magnetic dots could efficiently be used not only to enhance the flux pinning, but also to tune the nucleation of superconductivity [12].

Theoretical studies of individual mesoscopic superconductor/ferromagnet structures in the disk geometry were carried out in the framework of the Ginzburg-Landau formalism, with the emphasis on the vortex phases and transitions deep in the superconducting state [6, 8]. A mesoscopic superconducting $\mathrm{Al}$ disk with a perpendicularly magnetized magnetic dot was recently fabricated and its superconducting phase boundary $T_{c}(B)$ was measured $[3]$. These measurements demonstrated that the superconducting critical temperature is systematically higher when the applied magnetic field is parallel to the magnetization of the dot. However, in this sample the superconducting phase boundary seems to have been affected by the proximity effects between the superconducting disk and magnetic dot.

In this paper the nucleation of superconductivity in a mesoscopic superconducting Al loop, with a magnetic dot at the centre of the opening, is investigated. Given the spatial separation of the superconducting loop and magnetic dot, it is clear that the dot interacts with the loop exclusively through the stray field and proximity effects are absent. Therefore, any peculiar features in the $T_{c}(B)$ phase boundary can undoubtedly be attributed to the magnetic interaction between the superconducting condensate and the stray field of the dot.

The sample was prepared by electron beam lithography in three steps on a $\mathrm{SiO}_{2}$ substrate using positive PMMA950K and the corresponding co-PMMA electron beam resists. The lithography was carried out in a JEOL 5600 scanning electron micrograph modified for electron beam writing. The contacts pads, with the size of $500 \mu \mathrm{m}$, leads and alignment markers were patterned first and $5 \mathrm{~nm}$ of $\mathrm{Cr}$ and $30 \mathrm{~nm}$ of $\mathrm{Au}$ were thermally evaporated. Afterwards, the resist was removed with a lift-off procedure, using warm acetone and ultrasonic agitation. The sample was covered again with the e-beam resist. After the alignment, the mesoscopic loop and contacts were patterned. The designed width of the loop and contacts is $400 \mathrm{~nm}$, whereas the inner radius of the loop is $600 \mathrm{~nm}$. In order to avoid proximity effects, the mesoscopic contacts next to the loop are $22 \mu \mathrm{m}$ apart from the $\mathrm{Cr} / \mathrm{Au}$ leads. $35 \mathrm{~nm}$ of $\mathrm{Al}$ was thermally evaporated and a lift-off was carried out. In the final step, the sample was yet again covered with the e-beam resists. After a quite precise alignment procedure, magnetic dot with the radius of $200 \mathrm{~nm}$ was patterned. A magnetic dot consisting of $2.5 \mathrm{~nm}$ Pd buffer layer and 10 bilayers of $0.4 \mathrm{~nm}$ Co and $1 \mathrm{~nm} \mathrm{Pd}$ was subsequently evaporated. The resist was removed in a lift-off.

Fig. 11 shows an electron micrograph of the structure. The bright area at the centre is magnetic dot. The actual dimensions of the loop and magnetic dot were obtained from atomic force microscopy. The inner radius of the loop equals $R_{i}=0.55 \mu \mathrm{m}$, outer radius is equal to $R_{o}=1.05 \mu \mathrm{m}$, whereas the $\mathrm{Co} / \mathrm{Pd}$ magnetic dot has the radius of $r_{d}=0.27 \mu \mathrm{m}$. The mesoscopic contacts next to the loop, used for measuring voltage, are closer to the loop than initially designed. This effectively increases the outer radius of the loop, since the supercurrent can spread into the contacts, thereby affecting both the periodicity and background of the phase boundary [1].

The superconducting phase boundary $T_{c}(B)$ was obtained by four-point transport measurements in a cryogenic setup at temperatures down to $1.11 \mathrm{~K}$, applying the magnetic field perpendicularly to the sample surface. A transport

\footnotetext{
* Dusan.Golubovic@fys.kuleuven.ac.be
} 


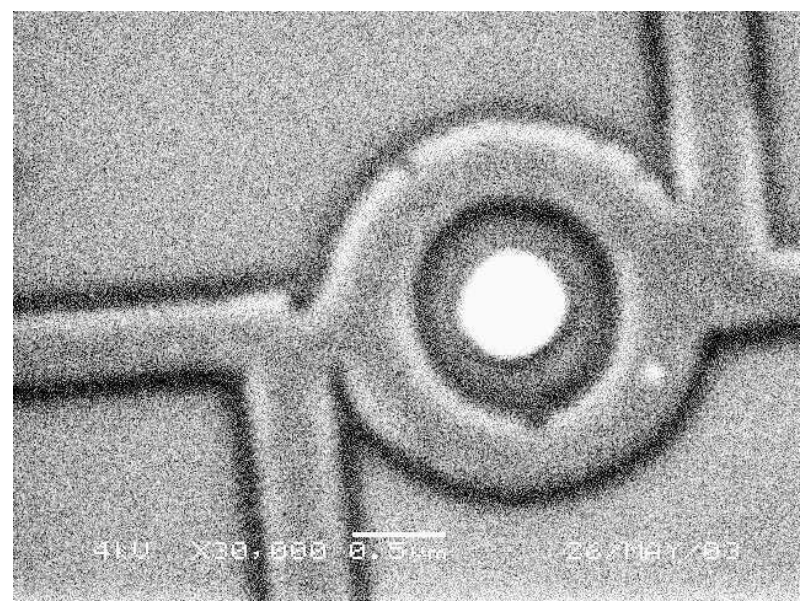

FIG. 1: A scanning electron micrograph of the structure.

current with the rms of $150 \mathrm{nA}$ and frequency $27.7 \mathrm{~Hz}$ was used. The response was measured by a PAR124A lockin amplifier. The phase boundary was determined resistively by sweeping the magnetic field at a very low rate, whilst keeping the temperature constant. $T_{c}(B)$ curve was afterwards extracted from the magnetoresistance using the resistive criterion $R_{n} / 2$, where $R_{n}$ is the resistance in the normal state. The temperature and field steps were $500 \mu \mathrm{K}$ and $5 \mu \mathrm{T}$, respectively, with the temperature stability $100 \mu \mathrm{K}$. In order to minimize the influence of possible temperature gradients, the temperature was being monitored by two independent temperature sensors placed at two different positions on the sample holder.

The resistance of the sample at room temperature is $6.6 \Omega$, the resistance in the normal state at low temperatures is $R_{n}=2.5 \Omega$, whereas the maximum critical temperature $T_{c m}$ is $1.3422 \mathrm{~K}$.

The multilayer $\mathrm{Co} / \mathrm{Pd}$ magnetic dot provides a perpendicular magnetization [12, 14]. The hysteresis loop in the

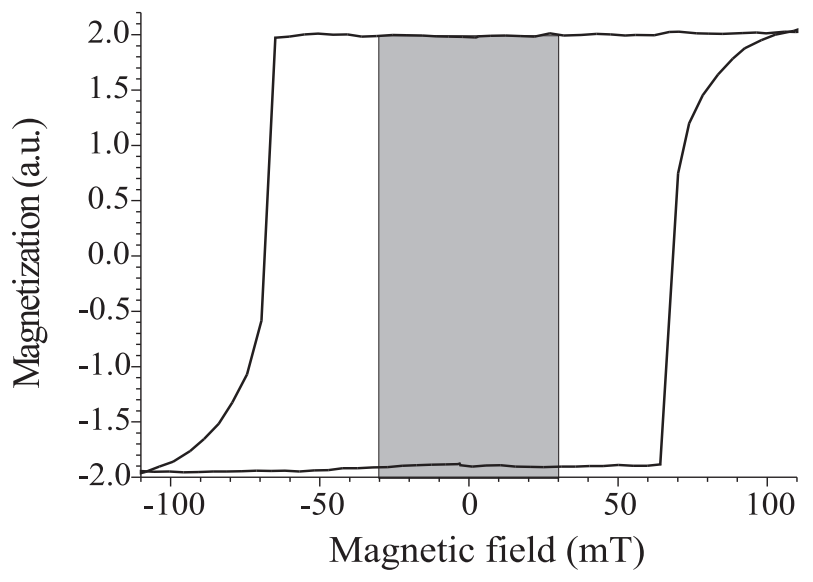

FIG. 2: The hysteresis loop of a co-evaporated $\mathrm{Co} / \mathrm{Pd}$ plane film obtained by the magneto-optical Kerr measurements at room temperature. The shaded area indicates the range of the applied magnetic field.

perpendicular magnetic field of the co-evaporated plane film with the same composition, obtained by the magnetooptical Kerr measurements at room temperature, is presented in Fig. 2 The shaded area indicates the range in which the applied magnetic field was varied in the experiment. The remanence is complete, whereas the coercive field is approximately $70 \mathrm{mT}$. When saturated, Co/Pd structures patterned at the sub-micrometre scale are in the single-domain state [12].

Fig. [3 shows the calculated stray field produced by a $\mathrm{Co} / \mathrm{Pd}$ magnetic dot, averaged over the thickness of the superconducting loop. The stray field is normalized to the magnetization of the magnetic dot. The darker shaded area shows the position of the magnetic dot, whereas the lighter shaded areas indicate the position of the superconducting loop. The arrow shows the direction of the magnetization $m$ of the dot. The stray field was calculated assuming a single-domain state of the magnetic dot. Prior to the measurements the dot was magnetized perpendicularly in 


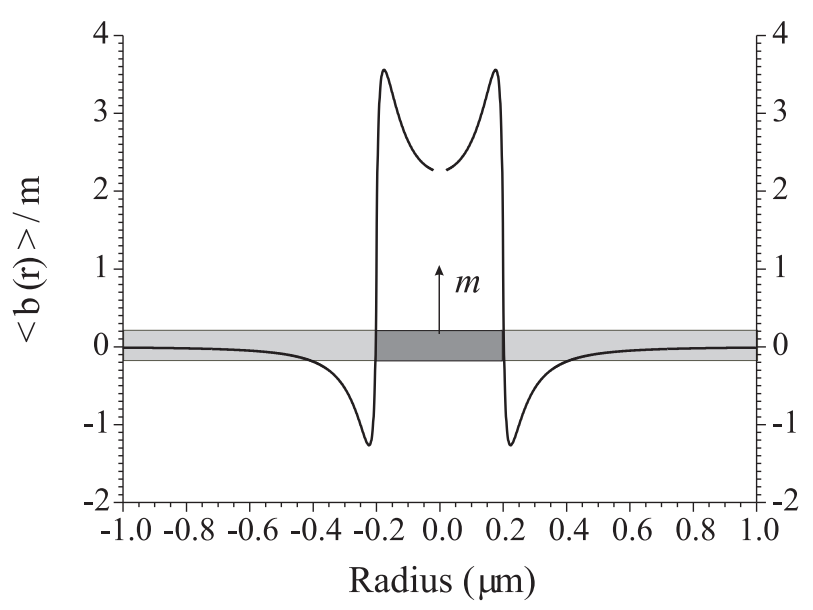

FIG. 3: The calculated stray field of the magnetic dot, averaged over the thickness of the superconducting loop. The stray field is normalized to the magnetization of the magnetic dot and was calculated assuming a single domain structure. The darker shaded area indicates the position of the dot, whereas the lighter shaded area shows the position of the superconducting loop. The arrow shows the direction of the magnetization.

$300 \mathrm{mT}$. As the applied magnetic field in our experiment never exceeded $30 \mathrm{mT}$ (Fig. 2), it has been assumed that the magnetization of the magnetic dot remains unaltered during the measurements [12].

The fluxes of the stray field (see Fig. 3) through the opening of the loop $(r<0.55 \mu \mathrm{m})$ and loop itself $(0.55<$ $r<1.05[\mu \mathrm{m}])$ have different orientations and, in qualitative terms, there should be a competition between the effects of these two fluxes on the superconducting condensate. Assuming that the loop is sufficiently big compared to the coherence length at zero temperature $\xi(0)\left(R_{i}, R_{o}>>\xi(0)\right.$, where $R_{i}$ and $R_{o}$ are the inner and outer radius of the loop, respectively) the loop is similar to a stripe at temperatures much lower than the maximum critical temperature the system can attain $T_{c m}$ (we will be referring to these temperatures as low temperatures). Clearly, the superconducting state of the stripe depends only upon the flux value within the stripe. In other words, at low temperatures the behaviour of the loop is governed by the flux through the loop and not by the flux in the opening of the loop. Therefore, at low temperatures the shift of the $T_{c}(B)$ phase boundary along the $B$-axis is expected to be in the direction of the dot magnetization. At temperatures close to the maximum critical temperature (we will be referring to these temperatures as high temperatures), the direction of the shift of the $T_{c}(B)$ phase boundary is a result of an interplay between the opposite fluxes. Note that the superconducting phase boundary does not depend on the local profile of the stray field in the opening of the loop, but on the total flux through the opening generated by magnetic dot.

To describe quantitatively the $T_{c}(B)$ phase boundary the Ginzburg-Landau theory has been used. The free energy of the superconducting structure can be expressed as

$$
F=2 \pi \int_{R_{i}}^{R_{0}} r d r\left[\alpha \psi+\beta \psi^{3}+\frac{\hbar^{2}}{4 m}\left(L-\frac{2 \pi}{\Phi_{0}} A\right) \psi^{2}+\frac{\hbar^{2}}{4 m}\left(\frac{d \psi}{d r}\right)^{2}\right]
$$

where $\psi$ is the modulus of the order parameter, $A$ is the vector potential, an integer number $L$ stands the winding number (vorticity) of the order parameter, $\Phi_{0}$ is the superconducting flux quantum, whereas $\alpha$ and $\beta$ represent the Ginzburg-Landau parameters. The integration is performed over the area of the loop. In Eq. (11) cylindrical symmetry of the order parameter near the phase transition to the normal state has been taken into account. As the thickness of sample is much smaller than $\xi(T)$ within the temperature range of interest, there is no modulation of the order parameter in the direction of the applied field. For this reason, the stray field can be averaged out over the thickness of the sample and the problem is reduced to the $1 \mathrm{D}$ case. Near the superconducting phase boundary the total magnetic field is equal to the sum of the stray field and an applied magnetic field. Therefore, the vector potential may be expressed as $A(r)=A_{s}(r)+B r / 2$, where $A_{s}(r)$ is the vector potential of the stray field and $B$ is an applied magnetic field. We represent $\psi(r)$ as a Fourier expansion within the range $R_{i} \leq r \leq R_{o}$ and consider the coefficients of the expansion the variational parameters. The values of these parameters can be calculated by a minimization of the free energy, yielding the solution of the full Ginzburg-Landau system of equations. The transition to the normal state corresponds to $\psi=0$. We have found that for our sample and experimental conditions, only the first term in the Fourier expansion is important, that is, $\psi$ is constant within the sample, whereas all other terms are negligibly small and practically do not affect the position of the phase boundary. 


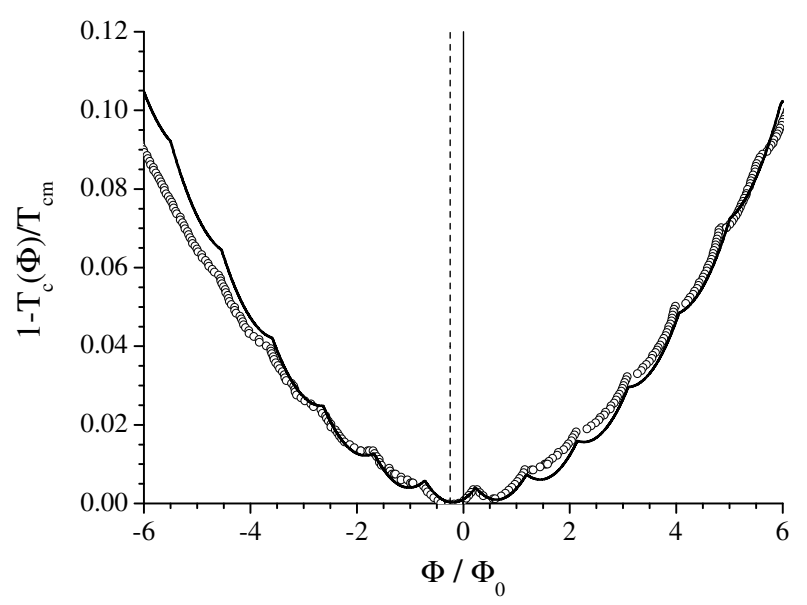

FIG. 4: The measured (open symbols) and calculated (solid line) superconducting phase boundary given as $1-T_{c}(B) / T_{c m}$ versus the normalized flux $\Phi / \Phi_{0}$. Here $T_{c m}$ is the maximum critical temperature and $\Phi_{0}$ is the superconducting flux quantum.

Fig. 4 presents the superconducting phase boundary, given as $1-T_{c}(B) / T_{c m}$ versus the normalized flux $\Phi / \Phi_{0}$. $T_{c m}$ is the maximum critical temperature and $\Phi_{0}$ is the superconducting flux quantum. Applied fields parallel to the magnetization of the dot are taken as positive and vice versa. The open symbols are experimental data, whereas the solid line corresponds to the theoretical curve for $R_{i}=0.55 \mu \mathrm{m}$, and $r_{d}=0.27 \mu \mathrm{m}$. The coherence length at zero temperature $\xi(T=0)$ has been used as a fitting parameter as it depends upon the preparation procedure and can show moderate variations even amongst samples prepared simultaneously. Due to the influence of the mesoscopic contacts, the outer radius has to be used as a fitting parameter, since the supercurrent is not confined only by the ring, but can well flow into the mesoscopic contacts, thus effectively increasing the outer radius. The best fit has been obtained for the coherence length at zero temperature $\xi(0)=96 \mathrm{~nm}$ and the outer radius $R_{o}=1130 \mathrm{~nm}$. The latter is quite close to the actual radius $R_{o}=1050 \mathrm{~nm}$.

The theoretical $T_{c}(B)$ curve is in a good agreement with the experimental data. The asymmetry, shift and periodicity of the experimental data have been reproduced well. The asymmetry and the shift can be explained by considering Eq. (11). From this equation it follows that the maximum critical temperature, for a fixed $L$, is attained when the applied field is equal to

$$
B=-\frac{4 \Phi_{0}}{\pi\left(R_{o}^{4}-R_{i}^{4}\right)} \int_{R_{i}}^{R_{o}} r^{2} d r\left(\frac{2 \pi A}{\Phi_{0}}-\frac{L}{r}\right)
$$

The corresponding critical temperature is

$$
1-\frac{T_{c}}{T_{c \infty}}=\frac{2 \xi(0)^{2}}{R_{o}^{2}-R_{i}^{2}}\left[\int_{R_{i}}^{R_{o}} r d r\left(\frac{2 \pi}{\Phi_{0}} A-\frac{L}{r}\right)^{2}-\frac{4}{R_{o}^{4}-R_{i}^{4}}\left(\int_{R_{i}}^{R_{o}} r^{2} d r\left(\frac{2 \pi}{\Phi_{0}} A-\frac{L}{r}\right)\right)^{2}\right]
$$

where $T_{c \infty}$ stands for the bulk critical temperature of Al. For the sake of simplicity, we assume that the stray field does not change considerably over the width of the loop. Denoting the average field in the opening of the loop and within the loop as $B_{o p}$ and $B_{l}$, respectively Eqs. (2) and (3) reduce to

$$
B=-B_{l}-\frac{2 \Phi_{0}}{\pi\left(R_{0}^{2}+R_{i}^{2}\right)}\left(\frac{\pi R_{i}^{2}\left(B_{l}-B_{o p}\right)}{\Phi_{0}}-L\right)
$$

and

$$
1-\frac{T_{c}}{T_{c \infty}}=\frac{2 \xi(0)^{2}}{R_{o}^{2}-R_{i}^{2}}\left(\frac{\pi R_{i}^{2}\left(B_{l}-B_{o p}\right)}{\Phi_{0}}-L\right)^{2}\left[\ln \left(\frac{R_{0}}{R_{i}}\right)-\frac{R_{o}^{2}-R_{i}^{2}}{R_{o}^{2}+R_{i}^{2}}\right]
$$

The maximum critical temperature $T_{c m}$ can be found by the minimization of the right-hand side of the Eq. (5) with respect to the integer $L$. Eq. (44) gives the shift of the $T_{c}(B)$ curve along the $B$-axis close to the $T_{c m}$, caused by the 
presence of an additional magnetic field. This shift is a sum of two contributions. The first term is just a compensation of the stray field $B_{l}$ within the loop. The second term describes the effect of the inhomogeneity of the stay field. This contribution behaves rather nontrivially due to the discreteness of $L$. For instance, when $0<\pi R_{i}^{2}\left(B_{o p}-B_{l}\right)<0.5 \Phi_{0}$, it follows from Eq. (5) that $L=0$, and the second contribution to the shift is negative. If the size of the dot and/or the intensity of the stray field are increased $0.5 \Phi_{0}<\pi R_{i}^{2}\left(B_{o p}-B_{l}\right)<\Phi_{0}, L$ switches to 1 , and the second contribution becomes positive. Physically, this means that it is energetically more favorable for the system to change the vorticity by 1 in order to decrease the modulus of the current in the loop. As a result, the current and the total shift, defined by Eq. (4), can change the sign. Thus, we may conclude, that the contribution to the shift, which is caused by the stray field inhomogeneity, is a periodic function of $B_{o p}-B_{l}$ and vanishes in the case of a homogeneous field, $B_{o p}=B_{l}$. The total shift of the $T_{c}(B)$ curve along the $B$-axis close to the $T_{c m}$ can be of both signs for the same orientation of magnetization of the dot.

Our calculations have shown that at low temperatures the shift of the phase boundary is controlled only by the average stray field within the loop and is equal to $-B_{l}$, which is in accordance with the preliminary qualitative analysis. If the additional magnetic field were homogeneous, the shifts of the $T_{c}(B)$ phase boundary for low and high temperatures would have the same direction. The inhomogeneity of the stay field gives rise to two different and mutually independent shifts of the phase boundary in the low and high temperature ranges, which, in turn, twist the whole superconducting phase boundary, as presented in Fig. [4

We would like to note that the discrepancy between the theoretical and the experimental data for low temperatures can be attributed to the effect of contacts, since they change the geometry of the sample, which cannot be entirely accounted for only by using the outer radius of the loop as a fitting parameter.

To summarize, we have fabricated a mesoscopic superconducting loop with a magnetic dot at the centre of the opening and investigated the onset of superconductivity in this structure by measuring the superconducting $T_{c}(B)$ phase boundary. The phase boundary has been found to be asymmetric with respect to the polarity of an applied magnetic field. The structure exhibits the maximum critical temperature for a finite value of the applied magnetic field, which is antiparallel to the magnetization of the magnetic dot. The theoretical superconducting phase boundary, obtained in the framework of the Ginzburg-Landau theory, is in a good agreement with the experimental data. It has been demonstrated that the inhomogeneous stray field shifts the $T_{c}(B)$ phase boundary along the $B$-axis independently in the low and high temperature ranges. In the limit of a thin loop with a large radius, these shifts are determined by the average value of the stray field in the opening of the loop and within the loop.

The authors would like to thank G. Rens for AFM measurements. This work has been supported by the Belgian IUAP, the Flemish FWO and the Research Fund K. U. Leuven GOA/2004/02 programmes, as well as by the ESF programme "VORTEX". W. V. P. acknowledges the support from the Research Council of the K.U. Leuven and DWTC.

[1] V. V. Moshchalkov et al., Handbook of Nanostructured Materials and Nanotechnology 3, ed H. S. Nalwa (Academic Press, San Diego, 2000).

[2] V. V. Moshchalkov, V. Bruyndoncx and L. Van Look, Connectivity and Superconductivity, ed J. Berger and J. Rubinstein, (Springer-Verlag, Berlin 2000.)

[3] D. S. Golubovic, W. V. Pogosov, M. Morelle and V. V. Moshchalkov, Appl. Phys. Lett., 83, 1593 (2003).

[4] V. L. Pokrovsky and H. Wei, cond-mat/0305153 2003.

[5] A Yu Aladyshkin et al., cond-mat/0305551 2003.

[6] M. V. Milošević, S. V. Yampolskii and F. M. Peeters, Phys. Rev. B, 66, 024515 (2002).

[7] M. V. Milošević, S. V. Yampolskii and F. M. Peeters, Phys. Rev. B, 66, 174519 (2002).

[8] M. V. Milošević and F. M. Peeters, cond-mat/0211547 2002.

[9] L. E. Helseth, Phys. Rev. B, 66, 104508 (2002).

[10] S. Erdin et al., Phys. Rev. Lett., 88, 1 (2002).

[11] M. J. Van Bael et al., Phys. Rev. Lett., 86, 1 (2001).

[12] M. Lange, M. J. Van Bael, Y. Bruynseraede and V. V. Moshchalkov, Phys. Rev. Lett., 90, 197006 (2003).

[13] M. J. Van Bael et al., Physica C, 332, 12 (2000).

[14] P. F. Carcia, J. Appl. Phys., 63, 10 (1988). 